\title{
Cutaneous Lupus Erythematosus: Current Treatment Options
}

\author{
Jenna K. Presto, $B S^{1,2}$ \\ Victoria P. Werth, $M D^{1,2, *}$
}

\author{
Address \\ ${ }^{1}$ Corporal Michael J. Crescenz Veterans Affairs Medical Center (Philadelphia), \\ Philadelphia, PA, USA \\ *,2Department of Dermatology, University of Pennsylvania, Perelman Center for \\ Advanced Medicine Suite 1-330A, 3400 Civic Center Boulevard, Philadelphia, \\ 19104, PA, USA \\ Email: werth@mail.med.upenn.edu
}

Published online: 2 February 2016

(C) Springer International Publishing AG (outside the USA) 2016

This article is part of the Topical Collection on Lupus

Keywords Cutaneous lupus erythematosus - Topical steroids - Topical calcineurin inhibitors - Antimalarials . Immunosuppressives · Cutaneous Lupus Erythematosus Disease Area and Severity Index (CLASI ${ }^{\text {TI'}}$ )

\section{Opinion statement}

Cutaneous lupus erythematosus (CLE) is a heterogeneous autoimmune skin disease that can present with or without systemic lupus erythematosus (SLE). Managing CLE is important in order to reduce potential and established areas of damage, as well as improve quality of life (QOL). Non-drug therapy should be used in every case, which includes photoprotection, smoking cessation, and avoiding drugs that can trigger or exacerbate the disease. Topical corticosteroids and topical calcineurin inhibitors are often used in addition to lifestyle changes. Antimalarials are first-line systemic therapies, with hydroxychloroquine being the drug of choice. Quinacrine can be added to hydroxychloroquine for greater efficacy in hydroxychloroquine-refractory patients, and chloroquine can be used in place of hydroxychloroquine in patients who are unable to tolerate hydroxychloroquine. Second-line therapies include oral retinoids, immunosuppressives, immunomodulators, biologics, and pulsed dye laser. Systemic steroids may be necessary when bridging therapies but should normally be avoided due to their side effects. There is a paucity of high quality evidence with regard to management of cutaneous lupus, making it challenging to determine an appropriate treatment in refractory cases. Trials on existing therapies as well as new therapies are necessary in order to better treat patients with CLE.

\section{Introduction}

Lupus erythematosus (LE) is a broad-spectrum disease which can manifest as cutaneous lupus erythematosus
(CLE) or multisystem organ disease with systemic lupus erythematosus (SLE). CLE is divided into subgroups, 
consisting of chronic cutaneous lupus erythematosus (CCLE), subacute cutaneous lupus erythematosus (SCLE), and acute lupus erythematosus (ACLE). CCLE can be further divided; discoid lupus erythematosus (DLE) and lupus erythematosus tumidus (LET) are the most common forms of CCLE, while lupus panniculitis, hypertrophic lupus, mucosal lupus, chilblain lupus, and lichenoid DLE are less common forms of CCLE.

The goal of cutaneous lupus treatment is to reduce potential and established areas of activity and damage. The Cutaneous Lupus Erythematosus Disease Area and Severity Index (CLASI $\left.{ }^{\mathrm{TN}}\right)$ is a scoring system that has been validated to assess for skin disease activity and damage in cutaneous lupus patients. It has demonstrated intrarater and interrater reliability in dermatologists and rheumatologists [1, 2]. This scoring system has been used in numerous trials and has been shown to correlate with quality of life measures and disease improvement with a 4-point or $20 \%$ decrease in CLASI ${ }^{\text {Tw }}$ activity score deemed clinically significant $[2,3]$.

Improving quality of life is an important factor in treatment. Quality of life in cutaneous lupus patients has been assessed using the Skindex 29+3 [4], a validated skin-specific quality of life instrument, and the SF-36 [5], a validated general health quality of life form [6]. Patients with cutaneous lupus have a worse quality of life (QOL) as compared to other dermatologic conditions, such as acne, non-melanoma skin cancer, and alopecia. Poor quality of life for CLE patients has been associated with female gender, generalized disease, severe disease, facial lesions, and younger age [7]. These QOL indicators have been found to be similar between geographic populations [8]. Antimalarial or antimetabolite treatment response leads to decreased emotion, functioning, and symptom score using the Skindex 29 $[9 \bullet]$. This suggests the impact of treatment on a patient's well-being.

Treatment of cutaneous lupus should be based on severity and type of cutaneous lupus. Prevention of new lesions is the first key step. Prevention includes photoprotection, smoking cessation, and removal of exacerbating drugs. Topical therapy is often initiated with these preventative measures. Antimalarials remain the first-line systemic therapy and other systemic agents are used for recalcitrant cases or in patients who are unable to tolerate the antimalarials.

\section{Lifestyle}

UVA and UVB irradiation has been shown to induce lesions in patients with cutaneous lupus [10]. It is suggested that UV protection can reduce UV-triggered CLE lesions by inhibiting an interferon-mediated inflammatory response. CD11c- and CD123-positive dendritic cells, a source of interferons (IFNs), as well as IFN-induced human protein MxA are increased in unprotected irradiated skin of CLE patients and decreased when protected by broad spectrum sunscreen [11]. Due to the effects of ultraviolet radiation (UVR), sunscreen has become a mainstay treatment in the prevention of new lesions. Sunscreen can be divided into two groups: chemical filters and physical blockers. With chemical filters, UV blocking ability depends on the chemical structure. The chemicals can provide UVA, UVB, or both UVA/UVB protection. Physical blockers, such as titanium dioxide and zinc oxide, block both UVA and UVB radiation. Commercial sunscreens are composed of a variety of chemical filters and/or physical blockers. It is important to select a sunscreen with active ingredients that block both UVA and UVB radiation [12]. Patients should also use a sufficient amount of sunscreen $\left(2 \mathrm{mg} / \mathrm{cm}^{2}\right)$ with the highest sun protection factor (SPF) as possible. In a randomized, double-blind study, it was found that SPF 85 was significantly better in providing sunburn protection as compared to SPF 50 [13]. In addition to using broad spectrum sunscreen, patients should be advised to avoid midday sun and wear long sleeved shirts, pants, and 
broad-brimmed hats. Sun exposure may also occur unexpectedly in a car since window glass is UVA permeable. Choosing tinted and laminated car windows can prevent UVA exposure and thus help prevent lesions [14]. Other unexpected sources of UVR are fluorescent light bulbs. Patients should use bulbs with the lowest UV emission to prevent significant cumulative damage [15].

Smoking cessation should be strongly encouraged in patients with cutaneous lupus. Studies have found that current smokers with CLE have a higher

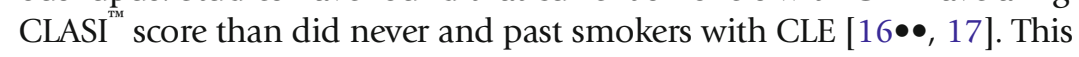
observation is consistent across geography, race, and ethnicity [17]. While some studies have suggested that smoking inhibits response to antimalarials [18], it is more likely that smoking has a direct effect on cutaneous lupus rather than a direct effect on the medications. In a study with 200 DLE patients treated with hydroxychloroquine, there was no difference in response rates between smokers and non-smokers [19]. In addition, it has been shown that mean CLASI ${ }^{\mathrm{Tm}}$ scores are higher in current compared to never and past smokers regardless of antimalarials, immunosuppressives, or corticosteroids use [17].

A thorough medication history should be taken in patients with SCLE as about one third of SCLE cases can be attributed to previous drug exposure. Drugs that have been implicated include terbinafine, tumor necrosis factor- $\alpha$ inhibitors, antiepileptics, and proton pump inhibitors. Since drug-induced SCLE is reversible once the drug is discontinued, it is important that patients with SCLE are appropriately screened [20].

\section{Pharmacologic treatment}

\section{Topical corticosteroids}

In conjunction with lifestyle changes, topical therapies are the first-line treatments for cutaneous lupus. Topical corticosteroids have been shown to be effective for all CLE subtypes. However, there is only one randomized controlled trial supporting their efficacy. This trial was a crossover study with 78 patients comparing fluocinonide $0.05 \%$ cream to hydrocortisone $1 \%$ cream. After 6 weeks of treatment, clearing or excellent improvement was seen in $27 \%$ of patients using fluocinonide and $10 \%$ in patients using hydrocortisone [21]. This significant difference suggests that a higher potency steroid is more effective than a lower potency steroid. Due to side effects associated with topical corticosteroids, including telangiectasias, acneiform eruption, and atrophy, the lowest potency required should be prescribed [22]. Topical corticosteroid selection should be based upon location of the lesion. Facial lesions should be treated with low potency steroids, such as $1 \%$ hydrocortisone butyrate, and stronger topical steroids should be used only for a few days and only on new or very active lesions. Trunk and arm lesions can be treated with a mid-potency topical, such as triamcinolone acetonide, while areas of thicker skin on the palms, soles, and scalp can be treated with potent steroids, such as clobetasol propionate [23].

Due to the side effects associated with topical steroids, topical calcineurin inhibitors, tacrolimus and pimecrolimus, have emerged as an alternative topical 
therapy. In a randomized, double-blind trial; 28 patients with different forms of CLE were treated with $0.1 \%$ tacrolimus ointment on one side of the face and $0.05 \%$ clobetasol propionate ointment on the other side of the face. Both tacrolimus and clobetasol significantly decreased disease activity with no difference between the two groups. However, $61 \%$ of patients developed telangiectasias on the clobetasol side [24]. Another randomized double-blind study compared $1 \%$ pimecrolimus cream with $0.1 \%$ betamethasone cream in 10 patients with DLE on the face. After 8 weeks of treatment, there was a decrease in clinical severity score of $86 \%$ in the pimecrolimus group and $73 \%$ in the betamethasone group. There was no significant difference between the groups, and no adverse effects were reported [25]. While topical calcineurin inhibitors are generally more expensive as compared to topical corticosteroids, their side effects are milder and include transient burning, erythema, and irritation [26]. Therefore, these studies suggest that topical calcineurin inhibitors are a more appropriate topical therapy for CLE lesions, especially in sensitive areas such as the face.

Antimalarials, including hydroxychloroquine, quinacrine, and chloroquine, are first-line systemic therapies often used in combination with lifestyle changes and topical treatments. Due to its lower incidence of side effects, hydroxychloroquine is considered the drug of choice. It has been shown that $60 \%$ of patients respond to hydroxychloroquine with others failing to respond or developing a toxicity requiring withdrawal [19]. These results are similar to another study showing a $55 \%$ response rate in patients started on hydroxychloroquine [23]. Localized disease and absence of SLE are associated with a response to hydroxychloroquine [19]. Hydroxychloroquine should be dosed at 200$400 \mathrm{mg} /$ day based on ideal body weight. Due to the risk of retinopathy, the American Academy of Ophthalmology recommends that patients receive a baseline eye examination and then annual screening after 5 years on hydroxychloroquine or sooner if there are risk factors [27].

When there is not a good response to hydroxychloroquine, quinacrine may be added. Quinacrine combined with hydroxychloroquine is associated with a $67 \%$ improvement rate in patients for whom hydroxychloroquine fails [23]. The recommended dose for quinacrine when combined with hydroxychloroquine is $100 \mathrm{mg} /$ day as this may lead to a more rapid improvement of skin lesions as compared to quinacrine dosed at $50 \mathrm{mg} /$ day [28]. Although quinacrine does not cause an additional risk for retinopathy when combined with hydroxychloroquine, it may cause bone marrow toxicity at higher doses over $100 \mathrm{mg} /$ day or yellowing of the skin [29]. Quinacrine is generally more expensive as compared to plaquenil since it is no longer commercially available and must be purchased at a compounding pharmacy that may not be covered by insurance.

Chloroquine may be used in place of hydroxychloroquine if the patient is not tolerating hydroxychloroquine. In a double-blind randomized control trial comparing clofazimine with chloroquine, $82.45 \%$ of patients on chloroquine had at least a $50 \%$ improvement in their 
lesions [30]. Although chloroquine is commonly used for cutaneous lupus, the use of clofazimine has been restricted due to the risk of antimicrobial resistance. Chloroquine should be dosed based on ideal body weight ( $\leq 3.5 \mathrm{mg} / \mathrm{kg} /$ day), usually at doses of $250 \mathrm{mg} /$ day 5 to 7 days/week. Since chloroquine is also associated with retinopathy, these patients should have a baseline eye examination with screening every 46 months. Due to an increased risk of retinopathy, hydroxychloroquine and chloroquine should not be used together. Like quinacrine, chloroquine must be purchased at a compounding pharmacy due to its lack of commercial availability.

Side effects shared by the antimalarials include xerosis, drug eruptions, urticaria, blue-gray skin hyperpigmentation, gastrointestinal upset, myopathy, cardiomyopathy, and rare central nervous system side effects [31].

\section{Systemic steroids}

Corticosteroids should normally be avoided due to their well-known side effects, including Cushing's syndrome, type 2 diabetes mellitus, and osteoporosis. However, it may be necessary to use steroids temporarily when bridging therapies that have a delayed onset of action. The recommended dosage is 0.5 to $1 \mathrm{mg} / \mathrm{kg} /$ day over 2 to 4 weeks followed by a taper. While SCLE has been shown to respond well to systemic steroids, DLE requires higher doses of steroids ( $>1 \mathrm{mg} / \mathrm{kg} / \mathrm{day})$ to achieve a response. Therefore, systemic steroids are not usually recommended for patients with DLE [22].

Oral retinoids are considered a second-line therapy for patients with CLE. Hydroxychloroquine $400 \mathrm{mg} /$ day was compared to acitretin $50 \mathrm{mg} /$ day in a trial with 58 subjects with CLE. Forty-six percent of patients showed marked or complete improvement in the acitretin group while $50 \%$ of patients showed marked or complete improvement in the hydroxychloroquine group. Although there was no significant difference between groups, there were more adverse events in the acitretin group, favoring the use the hydroxychloroquine [32]. Another study in three patients with different CLE subtypes received off-label systemic treatment with alitretinoin $30 \mathrm{mg}$ once daily. All three patients demonstrated good efficacy to the therapy [33]. Side effects, however, limit the use of oral retinoids. Oral retinoids are teratogenic and therefore contraception is necessary in women of child-bearing age before treatment as well as after treatment ( 1 month after isotretinoin discontinuation and 2 months after acitretin discontinuation) [29]. Retinoids may also result in dyslipidemia as well as elevated liver function tests [34].

Immunosuppressives can be used when patients are not responsive to antimalarials or are unable to tolerate them. Low-dose methotrexate has been shown to be as effective as chloroquine for cutaneous manifestations of SLE, which included SCLE, DLE, and malar rash [35]. The 
recommended dose is $7.5-25 \mathrm{mg}$ orally or subcutaneously once a week with folic acid supplementation. Side effects include gastrointestinal toxicity, bone marrow toxicity, nephrotoxicity, and hepatotoxicity [34]. Due to potential hepatotoxicity, patients should be advised against alcohol consumption during methotrexate usage.

Mycophenolate sodium and mycophenolate mofetil may be used in recalcitrant cases of CLE. In an open pilot study in ten patients with refractory SCLE, mycophenolate sodium led to a significant decrease in mean CLASI ${ }^{\text {TM }}$ score [36]. It has also been shown to be effective in combination with hydroxychloroquine in three patients who had antimalarial-resistant CLE. Doses of 1000 to $1500 \mathrm{mg} /$ day of mycophenolate were found to be effective in these patients with a mean response time of 5.6 weeks [37].

Azathioprine is another immunosuppressive which has demonstrated efficacy for DLE in several case series [39, 40]. Azathioprine is particularly useful in women who are pregnant as it does not affect fetal outcomes [38]. Due to the teratogenicity of several medications used in the treatment of CLE, azathioprine is often an appropriate alternative.

Dapsone has been successful in the treatment of cutaneous lupus and specifically bullous lupus. Three case series were combined to show a $55 \%$ improvement in CLE patients [39]. It was found that patients with bullous lupus had cessation of new blister formation within 1 to 2 days and healing of existing lesions within several days of dapsone usage dosed at $2 \mathrm{mg} / \mathrm{kg} /$ day [40]. Side effects of dapsone include agranulocytosis, methemoglobinemia, and hypersensitivity reactions. Due to the risk of hemolysis, dapsone is contraindicated in patients with glucose-6phosphate dehydrogenase deficiency [31].

Thalidomide has been used to treat refractory cases of cutaneous lupus. An observational study was performed in 60 patients with refractory CLE treated with thalidomide $100 \mathrm{mg}$ daily. Eighty-five percent of patients achieved complete response while $14 \%$ achieved partial response using the CLASI ${ }^{\mathrm{sin}}$. Following withdrawal of thalidomide, patients with DLE were more likely to relapse as compared to patients with SCLE. Paresthesias were reported by 11 patients with 5 of these patients having confirmed neuropathy with nerve conduction studies. Neuropathy was not found to correlate with treatment duration or dosage [41]. Although peripheral neuropathy and its teratogenic effects limit its usage, thalidomide should be considered as an effective medication for antimalarial-resistant CLE.

Lenalidomide is an analog of thalidomide with a lower risk of peripheral neuropathy. It is an immunomodulatory agent that targets the thalidomide-binding domain of cereblon, a protein part of a ubiquitin ligase complex [42]. This promotes the degradation of the transcription factors, aiolos and ikaros [43]. It has been suggested that ikaros plays a critical role in SLE pathogenesis due to its involvement in signal transduction and activation of transcription and IFN pathways [44•]. 


\section{Other treatments}

Lenalidomide has been successful in treating refractory CLE patients. In a case series with two severe recalcitrant DLE patients treated with lenalidomide, one patient showed no response, while the other patient had a sustained reduction in CLASI ${ }^{\text {Tw }}$ activity score [45]. Several open-label studies have also been performed. In one open-label study, four out of five patients demonstrated clinical improvement in their skin [46]. A larger open-label study with 15 patients found $86 \%$ of patients achieving a complete response, while another open-label study with 5 patients showed a mean CLASI $^{\text {TII }}$ activity score improvement from 21.4 at week 0 to 8.6 at week $12[47 \bullet, 48 \bullet$. Randomized controlled trials should be performed to better determine the safety and efficacy of lenalidomide.

\section{Biologics}

\section{Pulsed dye laser}

Rituximab is a monoclonal antibody to human CD20, which results in $\mathrm{B}$ cell death. Data suggests that there is a significant difference between subtype of cutaneous lupus and response to rituximab. In a study with 26 patients with different types of cutaneous lupus, 9 had a beneficial mucocutaneous response to rituximab at 6 months. However, none of the patients with CCLE responded, while $43 \%$ of the patients with ACLE (malar rash or widespread photoaggravated maculopapular rash) responded. In fact, new SCLE and CCLE lesions appeared during B cell depletion in patients with ACLE or no prior skin disease during the study, suggesting that SCLE and CCLE lesions are not B cell dependent

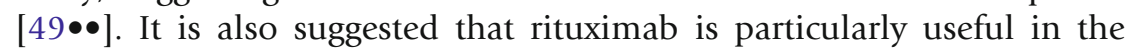
treatment of bullous lupus. In a case report, a patient with refractory bullous SLE had a complete skin response with no recurrence of lesions after two intravenous infusions of rituximab [50]. While additional studies are necessary, this case provides insight into the use of rituximab for bullous lupus.

Belimumab, a B lymphocyte stimulator-specific inhibitor, showed improvement in musculoskeletal and mucocutaneous organ domains in SLE after a post hoc analysis of data pooled from two phase III clinical trials [51]. Due to a limited treatment effect size, further studies would be needed to determine the efficacy of belimumab on cutaneous lupus.

Pulsed dye laser (PDL) has shown promising results in cases of refractory DLE. Raulin et al. demonstrated a median clearance rate of $70 \%$ in 9 patients with DLE treated with pulsed dye laser. Two of these patients developed hyperpigmentation following PDL, but this resolved at 4 and 5 months after therapy, respectively [52]. An open prospective study used PDL on 12 refractory patients with active DLE lesions. After comparing previous studies, they found that the best results were achieved using a fluence of $5.5 \mathrm{~J} / \mathrm{cm}^{2}$, spot size diameter of $7 \mathrm{~mm}$, and a treatment interval of 6 weeks. The study showed a significant decline in activity as measured by the CLASI ${ }^{\mathrm{Tm}}$ with no decline in damage scoring. One patient developed slight hyperpigmentation following treatment [53]. This demonstrates a viable option for patients with active, solitary DLE lesions who are refractory or unable to tolerate standard of care with topical therapies or antimalarials. 


\section{Emerging therapies}

\section{Spleen tyrosine kinase inhibitors}

Spleen tyrosine kinase (SYK) is involved in the regulation of inflammatory pathways. SYK inhibition has been shown to suppress established lupus skin and kidney disease as well as inhibit the development of lupus skin and kidney disease in lupus-prone mice. Clinical benefit for skin disease lasted for at least 8 weeks following discontinuation of the SYK inhibitor [54]. Further studies are being performed on humans to establish safety and efficacy.

Skin biopsy samples of DLE lesions have significantly increased interferon gamma signature as compared to normal skin [55]. A randomized, double-blind, placebo-controlled crossover study was performed with 16 patients to evaluate the effectiveness of AMG 811, a human IgG1 monoclonal antibody to IFN-gamma. Although the drug had a dosedependent inhibitory effect on IFN-gamma in the blood, there was no clinical improvement based on CLASI ${ }^{\text {TI }}$ scores and the intrasubject and intersubject heterogeneity between skin biopsies prevented any definitive conclusions from being made [56].

Therapies that target IFN-alpha have shown promising results for the treatment of SLE and cutaneous lupus. A phase 2b, randomized, doubleblind, placebo-controlled study was performed in 431 patients to evaluate the efficacy and safety of sifalimumab, an anti IFN-alpha monoclonal antibody. Significant improvements were seen in multiple clinical measures including the mucocutaneous domain. In patients with CLASI scores $\geq 10$, there was a higher percentage of patients with at least a 4point decrease in CLASI ${ }^{\mathrm{TM}}$ on sifalimumab $200 \mathrm{mg}(72.7 \%)$ and $1200 \mathrm{mg}(73.1 \%)$ compared to placebo (48.6\%) [57]. Another antiIFN-alpha monoclonal antibody, anifrolumab, has shown efficacy in a phase II, double-blind, placebo-controlled study. In patients with CLASI ${ }^{\text {Tu }}$ scores $\geq 10$, there was a higher percentage of patients with at least a $50 \%$ reduction in CLASI ${ }^{\mathrm{mix}}$ on anifrolumab $300 \mathrm{mg}(63.0 \%)$ compared to placebo $(30.8 \%)$ [58]. This suggests a potential therapy for CLE patients in the future.

\section{Anti-IL-6 monoclonal antibodies}

Anti-interleukin (IL-6) monoclonal antibodies have been approved to treat rheumatoid arthritis and systemic juvenile idiopathic arthritis. Recently, it has been used as an off-label treatment for autoimmune diseases, such as CLE, in which IL-6 plays a role in pathogenesis. Sirukumab, a human anti-IL-6 monoclonal antibody, was given in a phase I, randomized, double-blind, placebo-controlled study, which did not show any clinically significant changes from baseline for skin and systemic assessments. However, the study was not designed to evaluate 
for efficacy so further studies should be performed to assess for these endpoints [59].

Lenalidomide derivatives

Pharmacologic agents that are structurally similar to lenalidomide are under development. A lenalidomide derivative CC-220 has been shown to reduce aiolos and ikaros protein levels in B cells, $\mathrm{T}$ cells, and monocytes, which are proteins that are overexpressed in SLE peripheral blood. This derivative has been shown to inhibit anti-dsDNA and antiphospholipid autoantibody production in SLE peripheral blood [60]. Further studies are being performed to determine the safety and efficacy of lenalidomide derivatives.

\section{Trial design}

In the past 50 years, only one new drug has been approved for SLE and no drug has been specifically approved for CLE. This is not due to a shortage of patients, but rather, it is due to the challenges associated with trials on lupus. High placebo response rates due to background therapies make trial end points difficult to interpret. It is argued that newer treatment options are not necessary if standard treatments are resulting in high response rates. However, medications used for disease flares have serious side effects and are therefore not amenable to longterm use. This poses a critical need for new treatment with different trial designs. It may be beneficial to include subsets of patients with lower placebo response rates, such as refractory DLE or SCLE patients who do not respond well to background medications. This is exemplified by the Amgen trial, which showed no difference in response between AMG 811 and placebo in DLE patients [56]. This is in contrast to patients with ACLE who respond better to background medications making trial end points challenging to assess.

Cutaneous lupus patients are also often excluded from SLE studies even though there are no differences in skin disease between patients with cutaneous lupus only and patients with cutaneous lupus and SLE. This suggests a need for organ-specific trials in which skin may show a rapid response in effective trials. Due to the development of the CLASI ${ }^{\mathrm{TH}}$, a validated CLE outcome measure, it is now easier to evaluate the treatment of CLE-only disease as well as skin disease in SLE. The CLASI ${ }^{\text {Tu }}$ is currently used in almost all international studies that evaluate cutaneous lupus as an outcome. Other tools have not been fully validated $[61,62]$, present problems with difficult parameters to ascertain by dermatologists, let alone rheumatologists, such as differentiating infiltration versus hypertrophy, subcutaneous nodules, and surface area [61], and present limitations with the scope of disease measured [61, 62]

\section{Conclusion}

Cutaneous lupus erythematosus includes a broad spectrum of disease severity in association with or without systemic lupus erythematosus. 
Triggering factors such as UVR exposure, smoking, and implicated drugs should be avoided, and therefore, patient education is an important part of management. Topical corticosteroids and calcineurin inhibitors are first-line treatments. Antimalarials are the first-line systemic therapies, while refractory cases may benefit from combined therapies or secondline treatments. New therapies with better trial designs are necessary in order to find more effective treatments that minimize size effects.

\section{Compliance with Ethical Standards}

\section{Conflicts of Interest}

Jenna K. Presto declares that she has no conflicts of interest. Victoria P. Werth reports that she has received grants from Celgene, Biogen, Janssen, and Amgen; that she consults for Biogen, Medimmune, Celgene, Pfizer, Janssen, GSK, and Sanofi; and that she developed the CLASI, for which the copyright is owned by the University of Pennsylvania.

\section{Human and Animal Rights and Informed Consent}

With regard to the author's research cited in this paper, all procedures performed in studies involving human participants were in accordance with the ethical standards of the institutional and/or national research committee and with the 1964 Helsinki declaration and its later amendments or comparable ethical standards. In addition, all applicable international, national, and/or institutional guidelines for the care and use of animals were followed.

\section{References}

Papers of particular interest, published recently, have been highlighted as:

- Of importance

- Of major importance

1. Albrecht J, Taylor L, Berlin JA, Dulay S, Ang G, Fakharzadeh S, et al. The CLASI (Cutaneous Lupus Erythematosus Disease Area and Severity Index): an outcome instrument for cutaneous lupus erythematosus. J Invest Dermatol. 2005;125(5):889-94.

2. Jolly M, Kazmi N, Mikolaitis RA, Sequeira W, Block JA. Validation of the Cutaneous Lupus Disease Area and Severity Index (CLASI) using physician- and patientassessed health outcome measures. J Am Acad Dermatol. 2013;68(4):618-23.

3. Klein R, Moghadam-Kia S, LoMonico J, Okawa J, Coley C, Taylor L, et al. Development of the CLASI as a tool to measure disease severity and responsiveness to therapy in cutaneous lupus erythematosus. Arch Dermatol. 2011;147(2):203-8.

4. Chren MM, Lasek RJ, Flocke SA, Zyzanski SJ. Improved discriminative and evaluative capability of a refined version of Skindex, a quality-of-life instrument for patients with skin diseases. Arch Dermatol. 1997; 133(11):1433-40.
5. Ware Jr JE, Sherbourne CD. The MOS 36-item short-form health survey (SF-36). I. Conceptual framework and item selection. Med Care. 1992;30(6):473-83.

6. McHorney CA, Ware Jr JE, Raczek AE. The MOS 36Item Short-Form Health Survey (SF-36): ii. Psychometric and clinical tests of validity in measuring physical and mental health constructs. Med Care. 1993;31(3):247-63.

7. Klein R, Moghadam-Kia S, Taylor L, Coley C, Okawa J, LoMonico J, et al. Quality of life in cutaneous lupus erythematosus. J Am Acad Dermatol. 2011;64(5):84958.

8. Vasquez R, Wang D, Tran QP, Adams-Huet B, Chren $\mathrm{MM}$, Costner MI, et al. A multicentre, cross-sectional study on quality of life in patients with cutaneous lupus erythematosus. Br J Dermatol. 2013;168(1):145-53.

9. $\quad$ Chang AY, Ghazi E, Okawa J, Werth VP. Quality of life differences between responders and nonresponders in 
the treatment of cutaneous lupus erythematosus. JAMA

Dermatol. 2013;149(1):104-6.

This prospective study found that patients who responded to antimalarial or antimetabolite therapy had an improvement in skin-specific quality of life measures. This suggests the importance of treatment on quality of life.

10. Kuhn A, Sonntag M, Richter-Hintz D, Oslislo C, Megahed M, Ruzicka T, et al. Phototesting in lupus erythematosus: a 15-year experience. J Am Acad Dermatol. 2001;45(1):86-95.

11. Zahn S, Graef M, Patsinakidis N, Landmann A, Surber $\mathrm{C}$, Wenzel J, et al. Ultraviolet light protection by a sunscreen prevents interferon-driven skin inflammation in cutaneous lupus erythematosus. Exp Dermatol 2014;23(7):516-8.

12. Fett N, Werth VP. Systemic lupus erythematosus treatment: a guide to photoprotection. CML Rheum. 2010;29(2):33-41.

13. Russak JE, Chen T, Appa Y, Rigel DS. A comparison of sunburn protection of high-sun protection factor (SPF) sunscreens: SPF 85 sunscreen is significantly more protective than SPF 50. J Am Acad Dermatol. 2010;62(2):348-9.

14. Hampton PJ, Farr PM, Diffey BL, Lloyd JJ. Implication for photosensitive patients of ultraviolet A exposure in vehicles. Br J Dermatol.

2004;151(4):873-6.

15. Klein RS, Werth VP, Dowdy JC, Sayre RM. Analysis of compact fluorescent lights for use by patients with photosensitive conditions. Photochem Photobiol. 2009;85(4):1004-10.

16.• Piette EW, Foering KP, Chang AY, Okawa J, Ten Have TR, Feng R, et al. Impact of smoking in cutaneous lupus erythematosus. Arch Dermatol. 2012;148(3):317-22.

This prospective longitudinal cohort study 218 patients showed that current smokers had worse disease and worse quality of life as compared to never and past smokers.

17. Werth VP, Khamashta MA, Illei GG, Yoo S, Wang L, Greth W. Smoking is associated with more severe skin disease in subjects with moderate to severe systemic lupus erythematosus. [abstract]. Arthritis Rheum. 2013;65(Supp 10):2512.

18. Chasset F, Frances C, Barete S, Amoura Z, Arnaud L. Influence of smoking on the efficacy of antimalarials in cutaneous lupus: a meta-analysis of the literature. J Am Acad Dermatol. 2015;72(4):634-9.

19. Wahie S, Daly AK, Cordell HJ, Goodfield MJ, Jones SK, Lovell CR, et al. Clinical and pharmacogenetic influences on response to hydroxychloroquine in discoid lupus erythematosus: a retrospective cohort study. J Invest Dermatol. 2011;131(10):1981-6.

20. Gronhagen CM, Fored CM, Linder M, Granath F, Nyberg F. Subacute cutaneous lupus erythematosus and its association with drugs: a population-based matched case-control study of 234 patients in Sweden. Br J Dermatol. 2012;167(2):296-305.

21. Roenigk Jr HH, Martin JS, Eichorn P, Gilliam JN. Discoid lupus erythematosus. Diagnostic features and evaluation of topical corticosteroid therapy. Cutis. 1980;25(3):281-5.

22. Kuhn A, Ruland V, Bonsmann G. Cutaneous lupus erythematosus: update of therapeutic options part I. J Am Acad Dermatol. 2011;65(6):e179-93.

23. Chang AY, Piette EW, Foering KP, Tenhave TR, Okawa J, Werth VP. Response to antimalarial agents in cutaneous lupus erythematosus: a prospective analysis. Arch Dermatol. 2011;147(11):1261-7.

24. Tzung TY, Liu YS, Chang HW. Tacrolimus vs. clobetasol propionate in the treatment of facial cutaneous lupus erythematosus: a randomized, double-blind, bilateral comparison study. Br J Dermatol. 2007;156(1):191-2.

25. Barikbin B, Givrad S, Yousefi M, Eskandari F. Pimecrolimus $1 \%$ cream versus betamethasone 17 -valerate $0.1 \%$ cream in the treatment of facial discoid lupus erythematosus: a double-blind, randomized pilot study. Clin Exp Dermatol. 2009;34(7):776-80.

26. Sticherling M. Update on the use of topical calcineurin inhibitors in cutaneous lupus erythematosus. Biologics. 2011;5:21-31.

27. Marmor MF, Kellner U, Lai TY, Lyons JS, Mieler WF. Revised recommendations on screening for chloroquine and hydroxychloroquine retinopathy. Ophthalmology. 2011;118(2):415-22.

28. Cavazzana I, Sala R, Bazzani C, Ceribelli A, Zane C, Cattaneo R, et al. Treatment of lupus skin involvement with quinacrine and hydroxychloroquine. Lupus. 2009;18(8):735-9.

29. Winkelmann RR, Kim GK, Del Rosso JQ. Treatment of cutaneous lupus erythematosus: review and assessment of treatment benefits based on Oxford Centre for Evidence-based medicine criteria. J Clin Aesthet Dermatol. 2013;6(1):27-38.

30. Bezerra EL, Vilar MJ, da Trindade Neto PB, Sato EI. Double-blind, randomized, controlled clinical trial of clofazimine compared with chloroquine in patients with systemic lupus erythematosus. Arthritis Rheum. 2005;52(10):3073-8.

31. Okon LG, Werth VP. Cutaneous lupus erythematosus: diagnosis and treatment. Best Pract Res Clin Rheumatol. 2013;27(3):391-404.

32. Jessop S, Whitelaw DA, Delamere FM. Drugs for discoid lupus erythematosus. Cochrane Database Syst Rev. 2009;4:CD002954.

33. Kuhn A, Patsinakidis N, Luger T. Alitretinoin for cutaneous lupus erythematosus. J Am Acad Dermatol. 2012;67(3):e123-6.

34. Kuhn A, Ruland V, Bonsmann G. Cutaneous lupus erythematosus: update of therapeutic options part II. J Am Acad Dermatol. 2011;65(6):e195-213.

35. Islam MN, Hossain M, Haq SA, Alam MN, Ten Klooster PM, Rasker JJ. Efficacy and safety of methotrexate in articular and cutaneous manifestations of systemic lupus erythematosus. Int J Rheum Dis. 2012;15(1):62-8. 
36. Kreuter A, Tomi NS, Weiner SM, Huger M, Altmeyer P, Gambichler T. Mycophenolate sodium for subacute cutaneous lupus erythematosus resistant to standard therapy. Br J Dermatol. 2007;156(6):1321-7.

37. Sadlier M, Kirby B, Lally A. Mycophenolate mofetil and hydroxychloroquine: an effective treatment for recalcitrant cutaneous lupus erythematosus. J Am Acad Dermatol. 2012;66(1):160-1. author reply 1-2.

38. Saavedra MA, Sanchez A, Morales S, Angeles U, Jara LJ. Azathioprine during pregnancy in systemic lupus erythematosus patients is not associated with poor fetal outcome. Clin Rheumatol. 2015;34(7):1211-6.

39. Chang AY, Werth VP. Treatment of cutaneous lupus. Curr Rheumatol Rep. 2011;13(4):300-7.

40. Duan L, Chen L, Zhong S, Wang Y, Huang Y, He Y, et al Treatment of bullous systemic lupus erythematosus. J Immunol Res. 2015;2015:167064.

41. Cortes-Hernandez J, Torres-Salido M, CastroMarrero J, Vilardell-Tarres M, Ordi-Ros J. Thalidomide in the treatment of refractory cutaneous lupus erythematosus: prognostic factors of clinical outcome. Br J Dermatol. 2012;166(3):616-23.

42. Lopez-Girona A, Mendy D, Ito T, Miller K, Gandhi AK, Kang J, et al. Cereblon is a direct protein target for immunomodulatory and antiproliferative activities of lenalidomide and pomalidomide. Leukemia. 2012;26(11):2326-35.

43. Gandhi AK, Kang J, Havens CG, Conklin T, Ning $\mathrm{Y}$, $\mathrm{Wu} \mathrm{L}$, et al. Immunomodulatory agents lenalidomide and pomalidomide co-stimulate $\mathrm{T}$ cells by inducing degradation of $\mathrm{T}$ cell repressors ikaros and aiolos via modulation of the E3 ubiquitin ligase complex CRL4(CRBN.). $\mathrm{Br} \mathrm{J}$ Haematol. 2014;164(6):811-21.

44.• Hu SJ, Wen LL, Hu X, Yin XY, Cui Y, Yang S, et al. IKZF1: a critical role in the pathogenesis of systemic lupus erythematosus? Mod Rheumatol. 2013;23(2):205-9.

Ikaros, a protein targeted by lenalidomide, may play a critical role in SLE pathogenesis due to its involvement in signal transducers and activators of transcription and interferon (IFN) pathways.

45. Shah A, Albrecht J, Bonilla-Martinez Z, Okawa J, Rose $M$, Rosenbach $M$, et al. Lenalidomide for the treatment of resistant discoid lupus erythematosus. Arch Dermatol. 2009;145(3):303-6.

46. Braunstein I, Goodman NG, Rosenbach M, Okawa J, Shah A, Krathen M, et al. Lenalidomide therapy in treatment-refractory cutaneous lupus erythematosus: histologic and circulating leukocyte profile and potential risk of a systemic lupus flare. J Am Acad Dermatol. 2012;66(4):571-82.

47. Cortes-Hernandez J, Avila G, Vilardell-Tarres M, OrdiRos J. Efficacy and safety of lenalidomide for refractory cutaneous lupus erythematosus. Arthritis Res Ther. 2012;14(6):R265.
An open-label trial with lenalidomide demonstrated an 86\% complete response rate in patients with refractory cutaneous lupus. Lenalidomide has similar efficacy as compared to thalidomide but has a safer profile.

48. Okon L, Rosenbach M, Krathen M, Rose M, Propert K, Okawa J, et al. Lenalidomide in treatment-refractory cutaneous lupus erythematosus: efficacy and safety in a 52-week trial. J Am Acad Dermatol. 2014;70(3):583-4. In an open-label trial with five patients, lenalidomide use led to a significant decrease in CLASI ${ }^{\text {Tit }}$ from a mean of 21.4 at week 0 to a mean of 8.6 at week 12 .

49.• Vital EM, Wittmann M, Edward S, Md Yusof MY, Maclver H, Pease CT, et al. Brief report: responses to rituximab suggest B cell-independent inflammation in cutaneous systemic lupus erythematosus. Arthritis Rheumatol. 2015;67(6):1586-91.

This study showed that rituximab response is based on CLE subtype. New SCLE and CCLE lesions may be induced during B cell depletion.

50. Alsanafi S, Kovarik C, Mermelstein AL, Werth VP. Rituximab in the treatment of bullous systemic lupus erythematosus. J Clin Rheumatol. 2011;17(3):142-4.

51. Manzi S, Sanchez-Guerrero J, Merrill JT, Furie R, Gladman D, Navarra SV, et al. Effects of belimumab, a B lymphocyte stimulator-specific inhibitor, on disease activity across multiple organ domains in patients with systemic lupus erythematosus: combined results from two phase III trials. Ann Rheum Dis. 2012;71(11):1833-8.

52. Raulin C, Schmidt C, Hellwig S. Cutaneous lupus erythematosus-treatment with pulsed dye laser. $\mathrm{Br} \mathrm{J}$ Dermatol. 1999;141(6):1046-50.

53. Erceg A, Bovenschen HJ, van de Kerkhof PC, de Jong EM, Seyger MM. Efficacy and safety of pulsed dye laser treatment for cutaneous discoid lupus erythematosus. J Am Acad Dermatol. 2009;60(4):626-32.

54. Deng GM, Liu L, Bahjat FR, Pine PR, Tsokos GC. Suppression of skin and kidney disease by inhibition of spleen tyrosine kinase in lupus-prone mice. Arthritis Rheum. 2010;62(7):2086-92.

55. Jabbari A, Suarez-Farinas M, Fuentes-Duculan J, Gonzalez J, Cueto I, Franks Jr AG, et al. Dominant Th1 and minimal Th17 skewing in discoid lupus revealed by transcriptomic comparison with psoriasis. J Invest Dermatol. 2014;134(1):87-95.

56. Werth V, Fiorentino D, Cohen S, Fibenson D, Hansen C, Zoog S, et al. A phase I single-dose crossover study to evaluate the safety, toelrability, pharmacokinetics, pharmacodyanmics, and clinical efficacy of AMG 811 (anti-IFN-gamma) in subjects with discoid lupus erythematosus [abstract]. Arthritis Rheum. 2013;65(supp 10):1608.

57. Khamashta M, Merill JT, Werth VP, Furie R, K K, Illei GG et al. Safety and efficacy of sifalimumab, an antiIFN-alpha monoclonal antibody in a phase $2 \mathrm{~b}$ study of moderate to severe systemic lupus erythematosus. [abstract]. Arthritis Rheum. 2014;66:3531.

58. Furie R, Merill J, Werth V, Khamashta M, Kalunian K, Brohawn $\mathrm{P}$ et al. Anifrolumab, an anti-interferon alpha 
receptor monoclonal antibody, in moderate to severe systemic lupus erythematosus (SLE) [abstract]. Arthritis Rheumatol. 2015;67 suppl 10.

59. Szepietowski JC, Nilganuwong S, Wozniacka A, Kuhn A, Nyberg F, van Vollenhoven RF, et al. Phase I, randomized, double-blind, placebo-controlled, multiple intravenous, dose-ascending study of sirukumab in cutaneous or systemic lupus erythematosus. Arthritis Rheum. 2013;65(10):2661-71.

60. Schafer P, Ye Y, Wu L, Kosek J, Yang Z, Liu L, et al. The CRL4 cereblon E3 ubiquitin ligase modulator CC-220 induces degradation of the transcription factors aiolos and ikaros: immunomodulation in healthy volunteers and relevance to systemic lupus erythematosus [abstract]. Ann Rheum Dis. 2015;74:113.

61. Kuhn A, Meuth AM, Bein D, Amler S, Beissert S, Bohm M, et al. Revised Cutaneous Lupus Erythematosus Disease Area and Severity Index (RCLASI): a modified outcome instrument for cutaneous lupus erythematosus. Br J Dermatol. 2010;163(1):83-92.

62. Wahie S, McColl E, Reynolds NJ, Meggitt SJ. Measuring disease activity and damage in discoid lupus erythematosus. Br J Dermatol. 2010;162(5):1030-7. 